\title{
An amorphous titanium dioxide metal insulator metal selector device for ReRAM crossbar arrays with tunable voltage margin
}

Simone Cortese, Ali Khiat, Daniela Carta, Mark E. Light and Themistoklis Prodromakis.

Resistive RAM (ReRAM) crossbar arrays have become one of the most promising candidates for next-generation non volatile memories (NVMs). To become a mature technology, the sneak path current issue must be solved without compromising all the advantages that crossbars offer in terms of electrical performances and fabrication complexity. Here, we present an highly integrable access device based on nickel and substoichiometric amorphous titanium dioxide (TiO_\{2-x\}), in a metal insulator metal (MIM) crossbar structure. The high voltage margin of $3 \mathrm{~V}$, amongst the highest reported for monolayer selector devices, and the good current density of $104 \mathrm{~A} / \mathrm{cm} 2$ make it suitable to sustain ReRAM read and write operations, effectively tackling sneak currents in crossbars without compromising fabrication complexity in a 1 Selector 1 Resistor (1S1R) architecture. Furthermore, the voltage margin is found to be tunable by an annealing step without affecting the device's characteristics. 